\title{
Erratum to: Cone opsins and response of female chamois (Rupicapra rupicapra) to differently coloured raincoats
}

\author{
S. Raveh • W. F. D. van Dongen • C. Grimm • P. Ingold
}

Published online: 29 May 2012

(C) Springer-Verlag 2012

\section{Erratum to: Eur J Wildl Res (2012) DOI 10.1007/s10344-012-0629-Z}

The original version of this article inadvertently contained a mistake. The figure captions do not correspond to the correct figures. Below are the correct captions for each figure.

Fig. 1 Distribution of the chamois after the animals took flight from the salt lick (indicated by the black circle) after

The online version of the original article can be found at http://dx.doi.org/ 10.1007/s10344-012-0629-z.

\author{
S. Raveh $\cdot$ P. Ingold \\ Ethology and Nature Conservation, Department of Zoology, \\ University of Bern, \\ Länggassstrasse 27, \\ 3012 Berne, Switzerland \\ S. Raveh $(\bowtie) \cdot$ W. F. D. van Dongen \\ Konrad Lorenz Institute of Ethology, Department of Integrative \\ Biology and Evolution, University of Veterinary Medicine, \\ Vienna, Austria \\ e-mail: shirleyraveh@hotmail.com \\ C. Grimm \\ Lab for Retinal Cell Biology, Department of Ophthalmology, \\ University of Zurich, \\ Wagistrasse 14, \\ 8952 Schlieren, Switzerland \\ Present Address: \\ P. Ingold \\ Färichweg 1, \\ 3038 Kirchlindach, Switzerland
}

being exposed to a hiker wearing coloured coats on the hiking trail (dotted line). The various symbols represent that position of refuge of chamois exposed to red raincoats (white triangle), yellow raincoats (black square) and blue raincoats (grey triangle)

Fig. 2 Morphology of the chamois retina. a Semithin section of the outer retina. Asterisk photoreceptor inner segments resembling cones. ONL, outer nuclear layer; PIS, photoreceptor inner segments; $P O S$, photoreceptor outer segments; $R P E$, retinal pigment epithelium. b Magnification of an area shown in a. Arrow, rod outer segment with discs containing rhodopsin; arrowhead, cone outer segment with discs containing cone opsin. Abbreviations and symbols as in a. c Semithin section through all layers of the retina of chamois. TAP, tapetum; INL, inner nuclear layer; $G C L$, ganglion cell layer; $N F L$, nerve fibre layer. Other abbreviations as in a. Scale bars 50 (a), 25 (b) and $100 \mu \mathrm{m}(\mathbf{c})$

Fig. 3 Selective staining of cone photoreceptors in the chamois retina. Immunofluorescence of retinal flatmounts with peanut agglutinin (green) and with antibodies raised against short-wavelength cone opsin ( $\mathrm{JH} 455$, red $)$ (a) or middle-wavelength cone opsin ( $\mathrm{JH} 492$, red) (b), respectively. The orange colour indicates co-localization. White arrows correspond to cone outer segments that were positive for peanut agglutinin only. Red arrows correspond to cone outer segments that were positive for both peanut agglutinin and short- (a) or middlewavelength (b) cone opsins, respectively 